\title{
Misuse of Antimicrobial Agents in Neonatal Units: A Cross-Sectional Survey in Kuwait
}

\author{
Rima M.Z. Al-Sawan a Amrit L. Soni a Qusai Al-Saleha \\ Abla A. Al-Alfy ${ }^{b}$ Mazin Al-Essad, e Usha Rajaramuc \\ Lekshminarayanapuram V. Deverajan ${ }^{d, e}$
}

Departments of Pediatrics, a Farwaniya Hospital, bAdan Hospital, and ' Jahra Hospital, dDepartment of Neonatology, Maternity Hospital, and 'Faculty of Medicine,

Kuwait University, Kuwait

\section{Key Words}

Neonatal unit - Nosocomial infection . Antimicrobial agents · Misuse

\begin{abstract}
Objective: To evaluate the justification for the use of antimicrobial agents and to verify the possibility of its misuse among inpatient neonates. Methods: A cross-sectional 1-day study was conducted. Two questionnaires $A$ and $B$ relating to inpatient neonates were filled by registrars working in the four neonatal units of the main regional hospitals (Maternity, Farwaniya, Adan, and Jahra). The objectives of the study were not revealed to the registrars. Results: Of the 119 patients, 57 $(48 \%)$ were on antimicrobial agents, and the cultures yielded positive in 25/57 (44\%). The organisms were reported not to be susceptible to the antimicrobial agents in use in 19/25
\end{abstract}

\begin{tabular}{ll}
\hline KARGER & @ 1999 S. Karger AG, Basel \\
Fax +4161306 12 34 & 1011-7571/99/0082-0119\$17.50/0 \\
$\begin{array}{l}\text { E-Mail karger@karger.ch } \\
\text { www.karger.com }\end{array}$ & $\begin{array}{l}\text { Accessible online at: } \\
\text { http://BioMedNet.com/karger }\end{array}$
\end{tabular}

$(76 \%)$ of the cases, but it was continued in $4 / 19$ (21\%). Antimicrobial agents were also continued in another 9/32 (28\%) despite negative culture results. Conclusion: Misuse of antimicrobial agents was homogeneously observed at various levels in all neonatal units. This implies that urgent action should be taken to overcome this problem.

\section{Introduction}

Antimicrobial agents are very commonly used in the neonatal units as the clinical signs and symptom of sepsis in these patients can be very nonspecific, to a degree that infection must be considered in virtually all cases of neonatal distress [1]. Also despite advances in various aspects of neonatal medical practices, and the impoved overall survival, infections

Dr. Rima M.Z. Al-Sawa

PO Box 1757

22018 Salmiyah (Kuwait)

Fax 5727779 
remain a serious cause of morbidity and mortality [1-3]. Clinicians' awareness of this problem might have increased the tendency for excessive use of antimicrobial agents, hence almost all critically ill neonates receive antimicrobials during their hospital stay, close to $90 \%$ in some reported series $[4,5]$. This phenomenon has resulted in increasing resistance of nosocomial pathogens in the neonatal intensive care units all over the world [2-7]. Kuwait has been concerned about the issue of the misuse of antimicrobial agents. In the last two decades there have been several studies to investigate this misuse [810]. However, to the best of our knowledge, this matter has not been looked into in the neonatal population. Hence this cross-sectional study was conducted in the neonatal units of the four regional hospitals in Kuwait namely: Maternity, Farwaniya, Adan and Jahra Hospitals, in order to evaluate the appropriateness of the use of antimicrobial agents.

\section{Patients and Methods}

A questionnaire 'A' was distributed to the registrars working in the neonatal units of the four regional hospitals. The staff were requested to include information about all neonatal inpatients on one particular day (11th June 1996). The information included was general epidemiological data, birth weight and gestational age, perinatal events and risk factors related to infections. Specific questions were asked about the indications for starting an antimicrobial agent, i.e., if the patient was already started on it on that day. Based on the reported indications the patients were categorized into three groups: (1) suspected sepsis: if the patient had clinical evidence of sepsis; (2) prophylactic: if the patient was undergoing an invasive procedure such as ventilation, catheterization, or was on steroids, and (3) miscellaneous: if the patient did not have 'suspected sepsis' or 'prophylactic' indications. Another questionnaire ' $\mathrm{B}$ ' was distributed to be filled up after a 3-month period. This included information about results of cultures and susceptibility of isolates from the latest specimens collected before and/or on 11th June 1996 and the total number and duration of antimicrobials used after the availability of culture and susceptibility results. The aim of the study was not revealed to the doctors involved in collecting and recording the data.

The methods used for collecting blood, culturing organism(s), identification and antimicrobial susceptibility were identical in three units: Farwaniya, Adan and Jahra Hospitals. Samples were collected in Bactec Peds Plus Medium (Becton Dickinson Inc., Cockeysville, Md., USA) and incubated in the Bactec 9240 (Becton Dickinson). Vitek gram-negative identification and gram-positive identification cards (bioMerieux Vitek Inc., Hazelwood, Mo., USA) and analytical profile index strips (bioMerieux, Marcy L'Etoile, France) were used for identification of isolates, and Vitek cards (bioMerieux) were used for antimicrobial susceptibility testing $[11,12]$. In the Maternity Hospital isolates were identified by a standard manual and analytical profile index, and the organism susceptibility was determined by the Kirby-Bauer disc diffusion method [11,12].

Septicemia was diagnosed by a positive blood culture, meningitis by a positive cerebrospinal fluid culture, and pneumonia by clinical and radiological signs plus a positive endotracheal or nasopharyngeal culture. Urinary tract infection was diagnosed by positive urine culture with a single organism at greater than $10^{4}$ colony-forming units per milliliter.

$\chi^{2}$ test with Yates' correction was used for analyzing the statistical significance of various data; $p$ value $<0.05$ was considered as statistically significant.

\section{Results}

The population of Kuwait in the 1996 census was $1,753,981$ with a crude birth rate of 25.4 per 1,000 population. In 1996 , a total of 30,746 live births were delivered in the four regional hospitals included in this study. This accounts for $70 \%$ of the total national live births [13].

The study population consisted of 119 inpatients with a mean age of 15.1 days (range 1-116 days), mean birth weight $2,054 \mathrm{~g}$ (range $840-4,600 \mathrm{~g}$ ) and a mean gestational age of 36.4 weeks (range 26-42 weeks). Male:female ratio was $1.078: 1$. The nationality of parents was: Kuwaiti 70/119 (59\%), non-Kuwaiti 
Fig. 1. $A m p=A m p i c i l l i n ; A m k=$ amikacin; Gen = gentamicin; $\mathrm{Ctx}=$ cefotaxime; Van = vancomycin; Oth $=$ others.

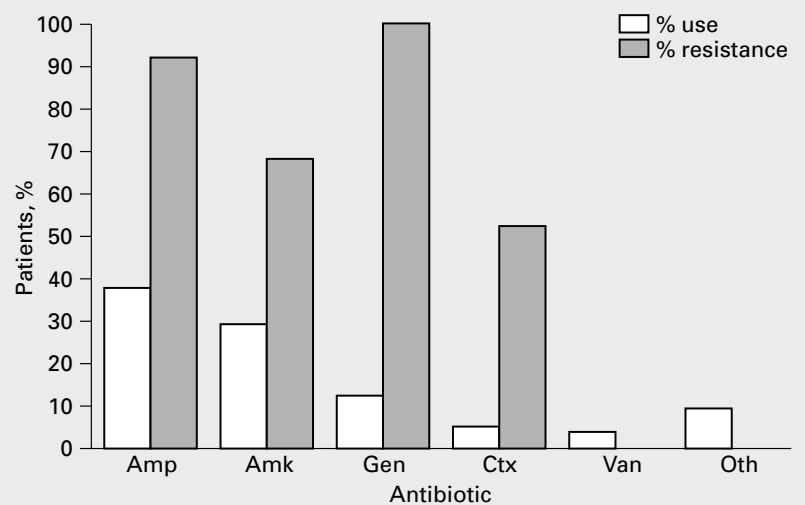

Arabs 26/119 (22\%) and non-Kuwaiti Asians 23/119 (19\%). The place of birth by hospital was as follows: Maternity 58/119 (49\%), Adan 23/119 (19\%), Farwaniya 20/119 (17\%) and Jahra 18/119 (15\%). The mode of delivery was vaginal route in $77 / 119(65 \%)$ and cesarean section in 42/119 (35\%). Significant birth asphyxia (Apgar score $<5$ at $5 \mathrm{~min}$ ) was present in $4 / 119$ cases (3\%). Premature rupture of membranes for more than $24 \mathrm{~h}$ was found in 13/119 cases (11\%).

Out of 119 patients, 57/119 (48\%) were receiving antimicrobial agents on the day of the study, but the total number of these used was 146 agents. This was because all the 57 patients were on at least two agents. In fact, $37 / 57(65 \%)$ patients were on two agents, $13 / 57(23 \%)$ were on three or four and $7 / 57$ $(12 \%)$ were on more than four antimicrobial agents. Ampicillin was the most commonly used antimicrobial agent $(55 / 146 ; 38 \%)$, followed by amikacin $(44 / 146 ; 30 \%)$, gentamicin $(19 / 146 ; 13 \%)$, cefotaxime $(8 / 146 ; 5 \%)$, vancomycin $(6 / 146 ; 4 \%)$, and others $(14 / 146$; $10 \%$ ): cloxacillin, metronidazole, ciprofloxacin, amphotericin B, piperacillin, teicoplanin and imipenem (fig. 1). The indications of starting antimicrobials were: suspected sepsis in $43 / 57$ cases $(75 \%)$, prophylactic in $13 / 57$ cases $(23 \%)$ and miscellaneous in $1 / 57$ case $(2 \%)$.

The analysis of the second questionnaire revealed that out of the 57 patients on antimicrobial agents, 25/57 (44\%) were found to have confirmed sepsis based on positive blood culture in $18 / 57$ cases (32\%), and other significant cultures in $7 / 57$ cases $(12 \%$, table 1). In confirmed sepsis gram-negative bacteria were isolated in $13 / 25$ cases (52\%), gram-positive in $8 / 25$ cases $(32 \%)$ and Candi$d a$ albicans in $4 / 25$ cases (16\%). There were $18 / 25$ cases $(72 \%)$ of septicemia, $2 / 25$ cases (8\%) of meningitis, $5 / 25$ cases $(20 \%)$ of pneumonia, and $1 / 25$ case $(4 \%)$ of urinary tract infection. There was an overlap in 1 patient who had septicemia and meningitis caused by the same organism (coagulase-negative staphylococcus). The majority of infections were late onset $19 / 25$ cases $(76 \%)$, but they were homogeneously distributed among different weight groups (table 2). An analysis of the studied risk factors for infection showed that 
Table 1. Distribution of cases with confirmed sepsis according to site of infection

\begin{tabular}{llllll}
\hline Organism & Septicemia & Meningitis & Pneumonia & UTI & Total \\
\hline Klebsiella pneumoniae & 3 & 0 & 1 & 0 & 4 \\
Pseudomonas aeruginosa & 3 & 0 & 1 & 0 & 4 \\
Enterobacter sp. & 2 & 0 & 0 & 0 & 2 \\
Proteus sp. & 1 & 0 & 0 & 0 & 1 \\
Flavobacterium sp. & 1 & 0 & 0 & 0 & 1 \\
Acinetobacter sp. & 1 & 0 & 0 & 0 & 1 \\
Staphylococcus aureus & 1 & 0 & 0 & 0 & 1 \\
MRSA & 1 & 0 & 2 & 0 & 3 \\
CNS & $2^{\mathrm{a}}$ & $1^{\mathrm{a}}$ & 1 & 0 & 3 \\
Streptococcus viridans & 1 & 0 & 0 & 0 & 1 \\
Candida albicans & 2 & 1 & 0 & 1 & 4 \\
\hline Total & 18 & 2 & 5 & 1 & 25 \\
\hline
\end{tabular}

MRSA = Methicillin-resistant S. aureus; CNS = coagulase-negative staphylococcus; UTI $=$ urinary tract infection .

a One patient had septicemia and meningitis.

Table 2. Distribution of positive cultures according to age of onset and birth weight among confirmed cases of sepsis

\begin{tabular}{lllllll}
\hline Organism & \multicolumn{2}{l}{ Onset $\leq 48 \mathrm{~h}$ old } & & \multicolumn{2}{l}{ Onset $>48 \mathrm{~h}$ old } & Total \\
\cline { 2 - 3 } & $\leq 1.5 \mathrm{~kg}$ & $>1.5 \mathrm{~kg}$ & & $\leq 1.5 \mathrm{~kg}$ & $>1.5 \mathrm{~kg}$ & \\
\hline Klebsiella & 2 & 0 & 2 & 0 & 4 \\
Pseudomonas & 1 & 1 & 1 & 1 & 4 \\
Other gram-negative organisms & 0 & 2 & & 1 & 2 & 5 \\
Staphylococcus & 0 & 0 & & 2 & 5 & 7 \\
Streptococcus & 0 & 0 & & 0 & 1 & 1 \\
Candida & 0 & 0 & & 3 & 1 & 4 \\
\hline Total & 3 & 3 & 9 & 10 & 25 \\
\hline
\end{tabular}

1 See table 1.

only ventilation and umbilical catheterization were significantly associated with confirmed sepsis (table 3 ).

In 19/25 (76\%) of the cases of confirmed sepsis, the antimicrobial agents being used would not have been effective according to the reported susceptibility of the organism isolated. Resistance was reported to be $100 \%$ for gentamicin, $92 \%$ for ampicillin, $69 \%$ for amikacin and $53 \%$ for cefotaxime (fig. 1). In the 19 cases where in vitro resistance was reported, $4 / 19$ cases $(21 \%)$ were continued on the same ineffective treatment and an additional antimicrobial agent was added. Out of 
Table 3. Significance of various risk factors in sepsis

\begin{tabular}{llll}
\hline Risk factor & $\begin{array}{l}\text { Sepsis } \\
(\mathrm{n}=25)\end{array}$ & $\begin{array}{l}\text { No sepsis } \\
(\mathrm{n}=32)\end{array}$ & $\mathrm{p}$ value \\
\hline Birth weight, $<1,500 \mathrm{~g}$ & 15 & 9 & n.s. \\
Gestation, $<33$ weight & 16 & 13 & n.s. \\
Male sex & 12 & 21 & n.s. \\
PROM, $>24 \mathrm{~h}$ & 4 & 1 & n.s. \\
Umbilical catheter & 8 & 2 & $<0.05$ \\
Mechanical ventilation & 7 & 1 & $<0.01$ \\
Intalipid & 8 & 6 & n.s. \\
\hline
\end{tabular}

PROM = Premature rupture of membranes. the 6 cases with effective empirical therapy, treatment was continued for more than 2 weeks in $4 / 6$ cases $(66 \%)$ even in the absence of meningitis or any other indication. In 9/32 cases $(28 \%)$ antimicrobial agents were continued despite negative culture results, presumably on clinical grounds.

\section{Discussion}

Resistance to antimicrobial agents is a serious problem in neonatal units all over the world [2-7]. Although the degree of the use of antimicrobials has shown a significant contribution to the emergence of resistant organisms, other factors intrinsic to the organisms themselves and to the critically ill patients may play a role $[2,14,15]$. As the symptomatology for sepsis in neonates is nonspecific, misuse of antimicrobials is much more probable [15-17]. In this cross-sectional study, we found that $57 / 119(48 \%)$ of inpatient neonates were receiving antimicrobial agents and the yield of positive cultures was 25/57 (44\%). This indicates that the decision of the clinicians to start the treatment was appropriate. However, we found that misuse/overuse exists at four different levels: (1) inappropriate choice of the empirical antimicrobials in $76 \%$ as evidenced by the susceptibility of the reported organisms; (2) prolonged use of appropriate antimicrobials in $66 \%$ of cases; (3) continuation of inappropriate antimicrobials in $21 \%$ of cases regardless of reports on in vitro resistance, and (4) persistent use of antimicrobials despite negative cultures in $28 \%$ of cases.

In a clinical situation, almost all sick neonates are started on empirical antimicrobials, usually a penicillin and an aminoglycoside. Ampicillin is usually the chosen penicillin as it covers Listeria monocytogenes which is a common isolate in early neonatal infection in Kuwait [18]. This combination should cover most of the vertically transmitted infections. The situation is different when a neonatal patient gets infected in the unit and the choice of antimicrobial agents must cover more serious nosocomial organisms such as Klebsiella pneumoniae, Pseudomonas aeruginosa, coagulase-negative staphylococci, and C. albicans infections. In recent studies in adult patients, resistance to cefotaxime was reported in $46 \%$ of K. pneumoniae and $73 \%$ of $P$. aeruginosa [19]. Similar resistance was reported to ceftazidime against a wide variety of gram-negative bacteria [15] and to vancomycin against enterococci [20]. In our study the isolated organisms were reported to be highly 
resistant to most of the commonly used antimicrobial agents, namely gentamicin, ampicillin, amikacin and cefotaxime (fig. 1). As most of the infections showed late onset (76\%), it might explain the high resistance of the reported organisms. Recent strains of multi-resistant Escherichia coli and $K$. pneumoniae producing an extended spectrum of $\beta$-lactamase have been reported in Farwaniya Hospital. The strains showed significantly reduced susceptibility to cefotaxime and were resistant to gentamicin and amikacin [2]. Based on this documented resistance and the fact that we are dealing with neonatal patients, the choice of which antimicrobial agent to use is difficult particularly in late-onset infections. This problem could be overcome by setting up policies stating which antimicrobial agents are suitable for a particular unit to match the susceptibility of organisms regularly isolated in the unit.

In this study, we found $27 \%$ of the suspected sepsis group and 69\% of the prophylactic group were confirmed cases of sepsis. Only mechanical ventilation $(\mathrm{p}<0.01)$ and indwelling catheters $(\mathrm{p}<0.05)$ were statistically significant factors associated with sepsis. Many studies report other factors such as low birth weight, prematurity, premature rupture membranes and male sex to be significantly associated with sepsis [1-7]. These factors were found to be not statistically significant in our study. Most neonatologists do not recommend prophylactic use of antimicrobial agents in many of these risk factors, but rather start them at the earliest definite indication of sepsis $[1,4,6,7,14-16]$. We strongly support this view. Good hand washing before and after all patient contact is reported to be the most important means of preventing nosocomial infections [21]. Hygienic hand disinfection will remove and/or kill most transient organisms [22]. However, awareness of this concept and its practices should be reinforced on all medical staff involved in patient care in neonatal units.

Although this was a cross-sectional oneday study with many limitations aimed at covering all the problems of neonatal infection and misuse of antimicrobial agents, it has thrown an insight onto some of the points which we should focus on, when trying to overcome these problems. Hence we propose the following recommendation: (1) Each unit must have a local policy for the use of antimicrobial agents in the treatment of early-onset and late-onset (nosocomial) infections based on continuous surveillance of the etiologic agents and their susceptibility patterns. (2) There should be a rigid control on the use of antimicrobials enforced by the senior medical staff. (3) Antimicrobial agents must be stopped as soon as possible, either after negative cultures, or a maximum of 2 weeks in uncomplicated sepsis. (4) Temptation to use antimicrobials despite reported resistance must be discouraged. (5) Compliance with hand hygiene to prevent nosocomial infections should be enforced. 


\section{References}

1 Roberton N: Infections in newborns; in Roberton NRC (ed): Textbook of Neonatology, ed 2. Edinburgh, Churchill Livingstone, 1992. pp 943-1005.

2 Al-Sawan R, Lionel J, West P, AlSaleh Q: Bacterial septicemia in Farwaniya neonatal unit-Kuwait. JKMA 1997;29:291-296.

3 Pegues DA, Arathoon EG, Samayoa B, Del Valle GT, Anderson RL, Riddle CF, O'Hara CM, Miller JM, Hill BC, Highsmith AK: Epidemic gramnegative sepsis in a neonatal intensive care unit in Guatemala. Am J Infect Control 1994;22:163-171.

4 Coovadia YM, Johnson AP, Bhana RH, Hutchinson GR, George RC, Hafferjee IE: Multiresistant Klebsiella pneumoniae in a neonatal nursery: The importance of infection control policies and procedures in the prevention of outbreaks. J Hosp Infect 1992;22:197-205.

5 Bryan CS, John JF Jr, Pai MS, Austin TL: Gentamicin vs cefotaxime for therapy of neonatal sepsis: Relationship to drug resistance. Am J Dis Child 1985;139:1086-1089.

6 Koutouby A, Habibullah J: Neonatal sepsis in Dubai, United Arab Emirates. J Trop Pediatr 1995;41: 177-180.

7 Daoud AS, Abuekteish F, Obeidat A, El-Nassir Z, Al-Rimawi H: The changing face of neonatal septicemia. Ann Trop Paediatr 1995;15:9396.
8 Thulesius O, Mark A, Kolleberg H, El-Hait SA, Kurain L: Patterns of antibiotic prescription in Kuwait. JKMA 1985; 19:135-145.

9 Elhag KM, Salah EA, El-Sabaa EM, Fakhry AF, Al-Ozairi SS: Antibiotic usage in teaching hospital in $\mathrm{Ku}-$ wait. KMAJ 1986;20:175-180.

10 Eldeen AS, Araj GF, Thulesius O, Skold O, Chugh TD, Salah EA, Elmardi NA: A comparison of antibiotics consumption and bacterial resistance patterns in Kuwait and Sweden. Acta Pathol Microbiol Immunol Scand 1988;3(suppl):59-62.

11 Baron EJ, Peterson LR, Finegold SM: Methods for the identification of etiological agents of infectious disease; in Diagnostic Microbiology, ed 9. St Louis, Mosby, 1994, pp 321-579.

12 NCCLS Antimicrobial Susceptibility Testing: Performance standards for antimicrobial disc susceptibility tests. NCCLS Document M2-A5. Villanova, 1993.

13 Mustafa MM: Annual Vital Statistics Report 1996. Vital Statistics Department, Ministry of Public Health, Kuwait.

14 Robinson A: Antibiotics and resistance: Hand in hand? CMAJ 1997; 150:927-928.
15 Toltzis P, Blumer JL: Antibioticresistant Gram negative bacteria in the critical care setting. Pediatr Clin North Am 1995;42:687-702.

16 Hensey OJ, Hart CA, Cooke RWI: Serious infections in a neonatal intensive care units: A two year survey. J Hyg 1985;95:289-297.

17 Hart CA: Klebsiella and neonates. J Hosp Infect 1993;23:83-86.

18 Lubani MM, Sharda DC, Al-Shab T, Sethi S: Neonatal listeriosis: A report of seven cases. Ann Trop Paediatr 1987;7:42-46.

19 Rotimi VO, Onyenefa PI, Banjo TO, Ogunsola FT, Adenuga A: Significance of antibiotics resistance amongst clinical bacterial isolate in Lagos. West Afr J Med 1994;13:8186.

20 Shay DK, Goldmann DA, Jrvis WR: Reducing the spread of antimicrobial-resistant microorganisms: Control vancomycin-resistant enterococci. Pediatr Clin North Am 1995; 42:703-716.

21 Sobayo EI, Haddad Q: Prevention of hospital-acquired infection. Postgrad Doctor M. East 1996;19:247252.

22 Casewell MW, Desai N: Survival of multiple resistant Klebsiella aerogenes and other Gram-negative bacilli on fingertips. J Hosp Infect 1983;4:350-360. 